\title{
Diagnostic characteristics, treatment patterns, and clinical outcomes for patients with advanced/metastatic medullary thyroid cancer
}

\author{
Rohan Parikh ${ }^{1 *}$, Lisa M. Hess' ${ }^{2}$ Elizabeth Esterberg ${ }^{1}$, Naleen Raj Bhandari² and James A. Kaye ${ }^{3}$
}

\begin{abstract}
Background: Medullary thyroid cancer (MTC) accounts for approximately 1.6\% of new cases of thyroid cancer. The objective of this study was to describe patient characteristics, biomarker testing, treatment patterns, and clinical outcomes among patients with advanced/metastatic MTC in a real-world setting in the United States and to identify potential gaps in the care of these patients.
\end{abstract}

Methods: Selected oncologists retrospectively reviewed medical records of patients aged $\geq 12$ years diagnosed with advanced MTC. Patients must have initiated $\geq 1$ line of systemic treatment for advanced/metastatic MTC between January 2013-December 2018 to be eligible. Patient characteristics, biomarker testing, and treatment patterns were summarized descriptively; progression-free survival (PFS) and overall survival (OS) were estimated using the KaplanMeier method.

Results: The 203 patients included in this study had a mean (SD) age of 52.2 (10.4) years; mean (SD) duration of follow-up from start of first-line treatment was 24.5 (16.0) months. Most patients (82.8\%) were initially diagnosed with stage IVA, IVB, or IVC disease. Among all patients, 121 (59.6\%) had testing for RET mutations, of whom 37.2\% had RETmutant MTC. The RET-mutation type was reported for 28 patients; the most common mutations reported were M918T (64.3\%) and C634R (32.1\%). Of the 203 patients, 75.9\% received only one line of systemic treatment for advanced disease, and $36 \%$ were still undergoing first-line therapy at the time of data extraction. Cabozantinib (30.0\%), vandetanib (30.0\%), sorafenib (17.2\%), and lenvatinib (4.9\%) were the most common first-line treatments. Among 49 patients who received second-line treatment, most received cabozantinib (22.4\%), vandetanib (20.4\%), lenvatinib (12.2\%), or sunitinib (12.2\%). Median PFS (95\% confidence interval [Cl]) from start of first- and second-line treatments was 26.6 months (20.8-60.8) and 15.3 months (6.6-not estimable [NE]), respectively. Median OS from initiation of first- and second-line treatment was 63.8 months (46.3-NE) and 22.4 months (12.4-NE), respectively.

Conclusions: For the treatment of advanced/metastatic MTC, no specific preference of sequencing systemic agents was observed in the first- and second-line settings. Considering the recent approval of selective RET inhibitors for patients with RET-mutant MTC, future research should investigate how treatment patterns evolve for these patients.

Keywords: RET, Medical record, Chart review, Retrospective, Observational, Real-world, United States survival

*Correspondence: rparikh@rti.org

${ }^{1}$ RTI Health Solutions, 3040 East Cornwallis Road, Research Triangle Park, NC 27709, USA

Full list of author information is available at the end of the article permits use, sharing, adaptation, distribution and reproduction in any medium or format, as long as you give appropriate credit to the original author(s) and the source, provide a link to the Creative Commons licence, and indicate if changes were made. The images or other third party material in this article are included in the article's Creative Commons licence, unless indicated otherwise in a credit line to the material. If material is not included in the article's Creative Commons licence and your intended use is not permitted by statutory regulation or exceeds the permitted use, you will need to obtain permission directly from the copyright holder. To view a copy of this licence, visit http://creativecommons.org/licenses/by/4.0/. The Creative Commons Public Domain Dedication waiver (http://creativeco mmons.org/publicdomain/zero/1.0/) applies to the data made available in this article, unless otherwise stated in a credit line to the data. 


\section{Background}

Medullary thyroid cancer (MTC) is a rare cancer evolving from neural crest-derived calcitonin-producing parafollicular C cells [1]. MTC accounts for $1.6 \%$ of all histologically confirmed incident thyroid tumors $(1,562 / 95,669$ cases) in the United States (US) [2]. A study of the US Surveillance, Epidemiology, and End Results (SEER) Program for cases diagnosed in 1994-2013 found that MTC accounted for $8.0 \%$ of all thyroid cancer-related deaths and $9.1 \%$ of age-adjusted thyroid cancer-related mortality during this period ${ }^{1}$ [3]. MTC can be either sporadic or hereditary, the latter occurring either with other endocrine neoplasms (multiple endocrine neoplasia [MEN] types $2 \mathrm{~A}$ and $2 \mathrm{~B}$ ) or alone (familial MTC). Most MTC cases are characterized by a mutation of the rearranged during transfection (RET) proto-oncogene, which can be either germline or somatic $[4,5]$. For example, an estimated $65 \%-90 \%$ of sporadic MTCs harbor somatic RET mutations [5-7], and autosomal dominant inheritance of an activating RET mutation causes hereditary MTC (both MEN2 syndromes and familial MTC) [8].

Approximately half of US patients with MTC are diagnosed with local disease [9]. The primary and curative treatment for most patients diagnosed with early-stage MTC comprises total thyroidectomy and neck dissection [10]. To treat patients with symptomatic advanced, progressive, or recurrent MTC, systemic therapies such as vandetanib, cabozantinib, and, for patients with $R E T$ mutant MTC, selpercatinib and pralsetinib are approved by the US Food and Drug Administration and included in national treatment guidelines [10]. With the availability of $R E T$-targeted therapies, genetic biomarker testing to identify $R E T$ alterations should be part of the standard of care for patients with advanced or metastatic MTC.

Evidence suggests that stage at diagnosis, presence and subtype of RET mutation, levels of biomarkers such as calcitonin and carcinoembryonic antigen (CEA), and type of systemic treatment may affect prognosis in advanced/metastatic MTC [11-15]. However, limited real-world evidence describes such patients in the US, their diagnostic and treatment patterns, and their clinical outcomes. This retrospective observational study evaluated the patterns of biomarker testing, treatments, and clinical outcomes among patients with advanced or metastatic MTC receiving routine clinical care.

\footnotetext{
${ }^{1}$ The reported age-adjusted annual incidence-based mortality estimates per 100,000 for MTC and for all thyroid cancers were 0.04 ( $95 \%$ confidence interval $[\mathrm{CI}], 0.03-0.04)$ and $0.44(95 \% \mathrm{CI}, 0.42-0.46)$, respectively, and $0.04 / 0.44=9.1 \%$.
}

\section{Methods}

\section{Study design overview}

An observational retrospective, medical record review of patients who had a confirmed diagnosis of advanced/ metastatic MTC was conducted. Participating oncologists (medical/clinical oncologists or hematologist/oncologists) who had treated $\geq 1$ patient with advanced MTC in the year before data abstraction, practiced for $\geq 3$ years after completion of formal training or board certification, and were the main decision-maker regarding treatment for their patients with advanced MTC abstracted demographic and clinical data into a customized, webbased case-report form. Oncologists were asked to select a quasi-random sample of their patients by abstracting medical records of those whose last names began with a randomly generated letter. Data abstraction occurred in April-May 2020. Data were then compiled into an analytic data set of deidentified patient-level data. RTI International's institutional review board (IRB) reviewed the study protocol and deemed the research, which was not considered human subjects research in accordance with the US Code of Federal Regulations (CFR) Sect. 45 CFR 46 , to be exempt from full IRB review.

\section{Study population}

Eligible patients had a diagnosis of histologically and/ or cytologically confirmed MTC or were initially diagnosed with or had progressed to having locally advanced or metastatic MTC (collectively referred to as "advanced MTC" hereafter). Patients were required to have initiated $\geq 1$ line of systemic anticancer treatment (single agent or combination) as their first therapy for advanced MTC (i.e., the eligible systemic therapy) between January 1,2013 , and December 31,2018 , be aged $\geq 12$ years at that time, and have a complete medical record covering all treatments after advanced MTC diagnosis. Decisions to initiate therapy were made by the treating physicians. The date of initiation of first-line therapy for advanced MTC was defined as the index date. Patients could be living or deceased at the time of record abstraction. Excluded patients had other malignant neoplasms before the index date (except MEN2-associated pheochromocytoma that had been resected or was documented to be stable; nonmelanoma skin cancer; in situ cervical cancer; or other cancer from which the patient had been disease free for $\geq 5$ years on the index date) or had participated in a clinical trial of an interventional drug as a first-line systemic treatment for advanced MTC.

Due to the retrospective, descriptive nature of this study, the targeted sample was not based on formal statistical considerations. Based on a feasibility assessment, a sample of approximately 200 patients across the US was planned. 


\section{Study measures}

In addition to patient characteristics, baseline information extracted from medical records included tumor stage at initial diagnosis, testing for potential germline or somatic mutations of special interest, and serum CEA and calcitonin levels. Systemic therapies received before and after advanced MTC diagnosis were recorded overall and by line of therapy. The sequence of regimens received for first- and second-line treatments was derived from the individual drug information for each line of therapy after advanced MTC diagnosis. Objective response (complete or partial response) to first- and second-line treatment was reported by each patient's oncologist. Criteria used by the treating clinician to assess response could include physical examination, performance status, nongenomic biomarker levels (calcitonin or CEA), imaging, or objective criteria (e.g., RECIST guidelines). Progression-free survival (PFS) and overall survival (OS) were estimated from the start of first- and second-line therapies. Clinician-defined disease progression, initiation of subsequent line of treatment, and death were considered progression events and were used to estimate PFS. Patient and tumor characteristics, treatments, and clinical outcomes were summarized for the overall study population and for the subgroup of patients with RET-mutant MTC.

\section{Statistical analyses}

All analyses were descriptive and were conducted using SAS (version 9.4, SAS Institute Inc., Cary, North Carolina). Time-to-event outcomes (OS and PFS from initiation of first-line and second-line therapies, respectively) were described using the Kaplan-Meier method. Subgroup analyses were conducted for patients with germline or somatic RET mutations. CEA and calcitonin levels were evaluated at advanced MTC diagnosis and during first- and second-line treatment. A post hoc mixed-model repeated-measures analysis accounting for within-patient correlation was conducted to evaluate improvement or decline in calcitonin levels and CEA levels during first-line therapy [16]. Time to decline of $\geq 50 \%$ from the calcitonin level and CEA level at initiation of the treatment line ( \pm 28 days) were each estimated from the mixed-model repeated-measures analysis.

\section{Results}

Seventy-five physicians (40 medical/clinical oncologists and 35 hematologist/oncologists) abstracted data from electronic medical records of a total of 203 patients with advanced MTC (per physician: mean, 2.8 patients [standard deviation [SD], 2.0; range, 1-6). The 75 participating physicians represented all geographic regions (Northeast, $n=19$ [25.3\%]; Midwest, $n=12$ [16.0\%]; South, $n=23$ [30.7\%]; and West, $n=21$ [28.0\%]) (Table 1). Most physicians $(n=59[78.7 \%])$ practiced in a cancer center or tertiary referral center $(n=34$ [45.3\%]) or a private hospital or clinic $(n=25[33.0 \%]) ; 13(17.3 \%)$ practiced in an academic or teaching hospital, and $3(4.0 \%)$ practiced in a nonteaching hospital setting. The mean (SD) number of years in practice, managing treatment of oncology patients since fully qualified, was 14.7 (5.7) years.

\section{Overall cohort of patients with advanced medullary thyroid cancer Patient and clinical characteristics}

At advanced MTC diagnosis, the mean (SD) age of patients included in this study was 52.2 (10.4) years; $58.6 \%$ of patients $(n=119)$ were female, and $66.0 \%$ $(n=134)$ were white (Table 2). Mean (SD) duration of follow-up was $24.5(16.0)$ years. Most patients $(n=168$; $82.8 \%$ ) had stage IV MTC (including IVA, IVB, and IVC) at initial diagnosis. Among the 141 patients who underwent evaluation of calcitonin level at advanced MTC diagnosis, 117 (83.0\%) had a known calcitonin level (mean [SD], 150.1 [138.9] pg/mL). Among the 108 patients who underwent CEA testing at advanced MTC diagnosis, 84 (77.8\%) had a known CEA level (mean [SD], $30.0[30.4] \mathrm{ng} / \mathrm{mL}$ ). Among the 173 patients whose performance status at advanced MTC diagnosis was known, $142(82.1 \%)$ had a performance status of $0 / 1$.

Most patients $(n=121 ; 59.6 \%)$ underwent biomarker testing for RET mutations (Table 3). Of the 45 (37.2\%) patients with known RET-mutant MTC, 25 (55.6\%) had $R E T$ mutations first identified before advanced MTC diagnosis, and 20 (44.4\%) had them first identified after advanced MTC diagnosis. Among patients with RET mutations, 18 (40\%) had M918T mutation, $9(20 \%)$ had C634R mutation, 1 (2\%) had C634G mutation, and the specific type of RET mutation was not known/documented for 17 (38\%) patients. Most patients had no hereditary clinical syndromes documented (169; 83.3\%); 15 patients $(7.4 \%)$ had a diagnosis of familial MTC, 8 (3.9\%) had MEN2A syndrome, and 5 (2.5\%) had MEN2B. The data collected did not distinguish between somatic and germline biomarker testing.

\section{Treatment patterns}

The mean time from advanced diagnosis to initiation of first-line therapy was $1.9(\mathrm{SD}=6.0)$ months (Table 4). Most patients $(n=154 ; 75.9 \%)$ received only one line of systemic anticancer therapy during the available followup time; 49 (24.1\%) received second-line therapy, and 4 $(2.0 \%)$ received third-line therapy. Overall, 73 patients (36\%) were receiving ongoing first-line treatment at data 
Table 1 Characteristics of Participating Physicians

\begin{tabular}{|c|c|c|}
\hline Total physician sample (N) & 75 & $100.0 \%$ \\
\hline \multicolumn{3}{|c|}{ Number of patients treated with advanced MTC in the last 12 months } \\
\hline Mean (SD) & $26.0(20.9)$ & \\
\hline Median (IQR) & $20.0(8.0-45.0)$ & \\
\hline \multicolumn{3}{|l|}{ Primary medical specialty, $(n, \%)$} \\
\hline Medical or clinical oncology (oncologist) & 40 & $53.3 \%$ \\
\hline Hematology-oncology & 35 & $46.7 \%$ \\
\hline \multicolumn{3}{|l|}{ Primary practice setting, $(n, \%)$} \\
\hline Cancer center/tertiary referral treatment center & 34 & $45.3 \%$ \\
\hline Academic/teaching hospital & 13 & $17.3 \%$ \\
\hline Other nonteaching hospital & 3 & $4.0 \%$ \\
\hline Private hospital or clinic & 25 & $33.3 \%$ \\
\hline \multicolumn{3}{|c|}{ Number of years in practice managing treatment of oncology patients since fully qualified } \\
\hline Mean (SD) & $14.7(5.7)$ & \\
\hline Median (IQR) & $15.0(10.0-18.0)$ & \\
\hline \multicolumn{3}{|l|}{ Geographic region of practice, $(n, \%)$} \\
\hline Northeast & 19 & $25.3 \%$ \\
\hline Midwest & 12 & $16.0 \%$ \\
\hline South & 23 & $30.7 \%$ \\
\hline West & 21 & $28.0 \%$ \\
\hline \multicolumn{3}{|c|}{ Number of patients for whom data was provided for this study } \\
\hline Mean (SD) & $2.8(2.0)$ & \\
\hline Median (IQR) & $2.0(1.0-4.0)$ & \\
\hline Min, Max & 1.0 & 6.0 \\
\hline
\end{tabular}

MTC medullary thyroid cancer, SD standard deviation

abstraction. The mean (SD) number of lines of therapy received was $1.3(0.5)$; mean (SD) total duration of systemic therapy was 12.0 (11.9) months. The most common first-line therapies received were cabozantinib $(n=61$, $30.0 \%)$, vandetanib $(n=61,30.0 \%)$, sorafenib $(n=35$, $17.2 \%)$, and lenvatinib $(n=10,4.9 \%)$; Table S-1 (Additional file 1) presents the most common regimens overall.

Figure 1 presents a Sankey chart of first- and secondline treatment sequences. At the end of study follow-up, $40 \%$ of patients had not received a subsequent line of treatment after discontinuing first-line treatment. Similar to first-line treatment, cabozantinib and vandetanib were received by similar proportions of patients in second-line therapy. Of the 61 patients (30\%) who received cabozantinib in the first line, 7 (11.5\%) received second-line vandetanib; of the 61 patients $(30 \%)$ who received vandetanib in the first line, 10 (16.4\%) received second-line cabozantinib.

During first-line treatment, 129 patients $(63.5 \%)$ were reported to have an objective response (i.e., complete or partial response); because of ongoing responses at data abstraction, median duration of objective response was not estimable for the 71 patients with known date of objective response (Table 5).

\section{Clinical outcomes}

For the overall study cohort, median PFS was 26.6 months (95\% confidence interval [CI], 20.8-60.8 months) from initiation of first-line therapy (Fig. 2A). Median OS was 63.8 months (95\% CI, 46.3 months-not estimable) from initiation of first-line therapy; survival estimates at 12, 36, and 60 months were $86.9 \%$ (95\% CI, 81.4\%-90.9\%), 63.5\% (54.0\%-71.5\%), and $60.5 \%$ (49.5\%-69.7\%), respectively (Fig. 3A). Disease-specific survival at 60 months was $68.3 \%$ (95\% CI, 58.9\%-76.0\%).

\section{Nongenomic biomarkers}

Calcitonin and CEA levels were evaluated per routine practice (at advanced diagnosis and/or during each treatment line). A total of 109 patients had 159 calcitonin evaluations at the initiation of first-line treatment ( \pm 28 days), and 83 patients had 328 calcitonin evaluations during the first-line treatment; 80 patients had 113 CEA evaluations at the initiation of first-line treatment ( \pm 28 days), and 45 patients had 113 CEA 
Table 2 Characteristics of Patients With Advanced Medullary Thyroid Cancer

\begin{tabular}{|c|c|c|c|c|}
\hline \multirow[b]{2}{*}{ Number of patients, $\mathbf{N}$} & \multicolumn{2}{|l|}{$\begin{array}{l}\text { All Patients, } \\
\mathrm{n}(\%)\end{array}$} & \multicolumn{2}{|c|}{$\begin{array}{l}\text { Patients With RET-Mutant } \\
\text { MTC, } \\
\text { n (\%) }\end{array}$} \\
\hline & 203 & $100.0 \%$ & 45 & $100.0 \%$ \\
\hline Age at advanced diagnosis of MTC & 203 & $100.0 \%$ & 45 & $100.0 \%$ \\
\hline Mean (SD) & $52.2(10.4)$ & & $46.6(9.7)$ & \\
\hline Median (IQR) & $53(44-59)$ & & $46(39-54)$ & \\
\hline \multicolumn{5}{|l|}{ Sex } \\
\hline Female & 119 & $58.6 \%$ & 26 & $57.8 \%$ \\
\hline Male & 84 & $41.4 \%$ & 19 & $42.2 \%$ \\
\hline \multicolumn{5}{|l|}{ Race $^{a}$} \\
\hline White & 134 & $66.0 \%$ & 24 & $53.3 \%$ \\
\hline Black/African American & 45 & $22.2 \%$ & 10 & $22.2 \%$ \\
\hline Asian, Native Hawaiian or other Pacific Islander & 18 & $8.9 \%$ & 6 & $13.3 \%$ \\
\hline Other $^{b}$ & 1 & $0.5 \%$ & 1 & $2.2 \%$ \\
\hline Unknown or not reported & 6 & $3.0 \%$ & 4 & $8.9 \%$ \\
\hline \multicolumn{5}{|l|}{ Ethnic origin } \\
\hline Hispanic or Latina/Latino & 25 & $12.3 \%$ & 5 & $11.1 \%$ \\
\hline Not Hispanic or Latina/Latino & 163 & $80.3 \%$ & 33 & $73.3 \%$ \\
\hline Unknown or not reported & 15 & $7.4 \%$ & 7 & $15.6 \%$ \\
\hline Total duration of follow-up, months ${ }^{c}$ & 203 & $100.0 \%$ & 45 & $100.0 \%$ \\
\hline Mean (SD) & $24.5(16.0)$ & & $28.8(18.0)$ & \\
\hline Median (IQR) & $21.1(15.0-28.5)$ & & $25.5(17.9-34.5)$ & \\
\hline \multicolumn{5}{|l|}{ Clinical stage at initial diagnosis } \\
\hline Stage II & 10 & $4.9 \%$ & 0 & $0.0 \%$ \\
\hline Stage III & 20 & $9.9 \%$ & 6 & $13.3 \%$ \\
\hline Stage IVA & 58 & $28.6 \%$ & 12 & $26.7 \%$ \\
\hline Stage IVB & 28 & $13.8 \%$ & 4 & $8.9 \%$ \\
\hline Stage IVC & 82 & $40.4 \%$ & 22 & $48.9 \%$ \\
\hline Unknown or not reported & 5 & $2.5 \%$ & 1 & $2.2 \%$ \\
\hline $\begin{array}{l}\text { Time from initial diagnosis to advanced diagnosis date (months), among patients } \\
\text { who were initially diagnosed with stage } \mathrm{I}, \mathrm{II}, \mathrm{III}(\mathrm{n}, \%)\end{array}$ & 30 & $14.8 \%$ & 6 & $13.3 \%$ \\
\hline Mean (SD) & $26.3(33.0)$ & & $37.5(64.0)$ & \\
\hline Median (IQR) & $14.1(8.2-28.8)$ & & $12.6(10.1-17)$ & \\
\hline Site(s) of local extension or metastasis at advanced MTC diagnosis ${ }^{\mathrm{a}}$ & 203 & $100 \%$ & 25 & $100 \%$ \\
\hline Distant lymph nodes & 111 & $54.7 \%$ & 20 & $44.4 \%$ \\
\hline Bone & 69 & $34.0 \%$ & 18 & $40.0 \%$ \\
\hline Brain & 14 & $6.9 \%$ & 6 & $13.3 \%$ \\
\hline Liver & 56 & $27.6 \%$ & 19 & $42.2 \%$ \\
\hline Local structures (e.g., muscles, larynx, trachea, esophagus, or large vessels) & 58 & $28.6 \%$ & 12 & $26.7 \%$ \\
\hline Lung/pleura & 81 & $39.9 \%$ & 15 & $33.3 \%$ \\
\hline Performance status at advanced MTC diagnosis $\left( \pm 30\right.$ days) ${ }^{d}$ & 173 & $85.2 \%$ & 42 & $93.3 \%$ \\
\hline 0 or 1 & 142 & $82.1 \%$ & 36 & $85.7 \%$ \\
\hline $2-4$ & 31 & $18.0 \%$ & 6 & $14.3 \%$ \\
\hline Calcitonin level evaluated at advanced MTC diagnosis ( \pm 30 days) $(\mathrm{pg} / \mathrm{mL})(\mathrm{n}, \%)^{\mathrm{e}}$ & 141 & $69.5 \%$ & 31 & $68.9 \%$ \\
\hline Calcitonin level known (n, \%) & 117 & $83.0 \%$ & 23 & $74.2 \%$ \\
\hline Mean (SD) & $150.1(138.9)$ & & $139.2(138.6)$ & \\
\hline Median (IQR) & $110.0(22.0-210.0)$ & & $100.0(18.0-200.0)$ & \\
\hline Calcitonin evaluated but level unknown or not reported ( $n, \%)$ & 24 & $17.0 \%$ & 8 & $25.8 \%$ \\
\hline CEA test performed at advanced MTC diagnosis ( \pm 30 days) $(\mathrm{ng} / \mathrm{mL})(\mathrm{n}, \%)^{f}$ & 108 & $53.2 \%$ & 26 & $57.8 \%$ \\
\hline CEA level known $(n, \%)$ & 84 & $77.8 \%$ & 14 & $53.8 \%$ \\
\hline
\end{tabular}


Table 2 (continued)

\begin{tabular}{|c|c|c|c|c|}
\hline \multirow[b]{2}{*}{ Number of patients, $\mathrm{N}$} & \multicolumn{2}{|l|}{$\begin{array}{l}\text { All Patients, } \\
\text { n (\%) }\end{array}$} & \multicolumn{2}{|c|}{$\begin{array}{l}\text { Patients With RET-Mutant } \\
\text { MTC, } \\
\mathrm{n}(\%)\end{array}$} \\
\hline & 203 & $100.0 \%$ & 45 & $100.0 \%$ \\
\hline Mean (SD) & $30.0(30.4)$ & & $27.5(28.2)$ & \\
\hline Median (IQR) & $18.3(3.7-50.0)$ & & $19.3(6.8-30.0)$ & \\
\hline CEA tested but level unknown or not reported $(n, \%)$ & 24 & $22.2 \%$ & 12 & $46.2 \%$ \\
\hline
\end{tabular}

CEA carcinoembryonic antigen, ECOG Eastern Cooperative Oncology Group, IQR interquartile ratio, MTC medullary thyroid cancer, RET rearranged during transfection, $S D$ standard deviation

${ }^{\text {a }}$ Multiple responses allowed; rows will add up to greater than $100 \%$

${ }^{\mathrm{b}}$ Reported as mixed race

' Length of follow-up is the duration of time between the date of initiation of first-line systemic therapy and death or end of patient record

${ }^{d}$ Karnofsky score were converted to the ECOG scale for 29 patients (14.3\%) [17]

${ }^{\text {e }}$ Calcitonin normal range: $<10 \mathrm{pg} / \mathrm{mL}$

${ }^{\mathrm{f}}$ CEA test normal range: $<2.5 \mathrm{ng} / \mathrm{mL}$

Table 3 RET-Mutation Testing and Diagnosis of Hereditary Medullary Thyroid Cancer Syndromes

\begin{tabular}{|c|c|c|}
\hline & $\mathbf{N}$ & $\%$ \\
\hline Total patient sample $(\mathrm{N})$ & 203 & $100.0 \%$ \\
\hline \multicolumn{3}{|l|}{ Tested for germline or somatic RET mutation at any time $(n, \%)$} \\
\hline Yes & 121 & $59.6 \%$ \\
\hline No/unknown & 82 & $40.4 \%$ \\
\hline \multicolumn{3}{|l|}{ Biomarker testing at or after initial diagnosis of MTC } \\
\hline $\begin{array}{l}\text { Patients tested for potential germline and/or somatic mutations at or after initial diagnosis of MTC ( } \mathrm{n} \text {, } \\
\%)\end{array}$ & 95 & $46.8 \%$ \\
\hline Patients with RET-mutant MTC (n, \%) & 45 & $37.2 \%$ \\
\hline RET mutation identified before initial MTC diagnosis & 25 & $55.6 \%$ \\
\hline RET mutation identified after initial MTC diagnosis & 20 & $44.4 \%$ \\
\hline Type of RET mutation among patients with RET-mutant MTC $(\mathrm{n}, \%)$ & 45 & $37.2 \%$ \\
\hline M918T & 18 & $40.0 \%$ \\
\hline C634R & 9 & $20.0 \%$ \\
\hline C634G & 1 & $2.2 \%$ \\
\hline Unknown & 17 & $37.8 \%$ \\
\hline \multicolumn{3}{|l|}{ Diagnosis of hereditary MTC syndromes at any time } \\
\hline MEN2A & 8 & $3.9 \%$ \\
\hline MEN2B & 5 & $2.5 \%$ \\
\hline Familial MTC & 15 & $7.4 \%$ \\
\hline No hereditary MTC syndrome diagnosed & 169 & $83.3 \%$ \\
\hline Unknown/not reported & 6 & $3.0 \%$ \\
\hline
\end{tabular}

MEN2A multiple endocrine neoplasia type 2A, MEN2B MEN type 2B, MTC medullary thyroid cancer; RET rearranged during transfection

${ }^{a}$ The data collection form did not distinguish between germline and somatic testing

evaluations during the first-line treatment. Regression modeling showed that calcitonin levels generally decreased during first-line treatment, with an estimated time to reach a decrease of $\geq 50 \%$ in calcitonin level occurring 8.4 months after treatment initiation (Fig. 4A). Similarly, CEA levels generally decreased during first-line treatment, with an estimated time to reach a decrease of $\geq 50 \%$ in CEA level occurring after 17.7 months (Fig. 4B).

\section{Patients with RET-mutant medullary thyroid cancer}

The 45 patients with RET mutations had a mean (SD) age of 46.6 (9.7) years, and most ( $n=38 ; 84.5 \%)$ had stage IV disease at advanced MTC diagnosis (Table 2). Among the 
Table 4 Systemic Therapy Patterns Among Patients With Advanced Medullary Thyroid Cancer

\begin{tabular}{|c|c|c|c|c|c|c|c|c|}
\hline \multirow[b]{3}{*}{ Number of patients initiating treatment $(\mathrm{N}, \%)$} & \multicolumn{4}{|c|}{ All MTC Patients } & \multicolumn{4}{|c|}{ Patients With RET-Mutant MTC } \\
\hline & \multicolumn{2}{|c|}{ First-line Therapy } & \multicolumn{2}{|c|}{ Second-line Therapy } & \multicolumn{2}{|c|}{ First-line Therapy } & \multicolumn{2}{|c|}{ Second-line Therap } \\
\hline & 203 & $100.0 \%$ & 49 & $100.0 \%$ & 45 & $100.0 \%$ & 13 & $100.0 \%$ \\
\hline Performance status at start of treatment ${ }^{\mathrm{a}},(\mathrm{n}, \%)$ & 138 & $68.0 \%$ & 35 & $71.4 \%$ & 30 & $66.7 \%$ & 9 & $69.2 \%$ \\
\hline 0 or 1 & 112 & $81.2 \%$ & 20 & $57.1 \%$ & 25 & $83.3 \%$ & 6 & $66.6 \%$ \\
\hline $2-4$ & 26 & $18.8 \%$ & 15 & $42.9 \%$ & 5 & $16.7 \%$ & 3 & $33.3 \%$ \\
\hline $\begin{array}{l}\text { Time from advanced MTC diagnosis to first line of } \\
\text { treatment and time between first and second line } \\
\text { of treatment, among patients who discontinued } \\
\text { first line of treatment ( } n, \%)\end{array}$ & 203 & $100.0 \%$ & 49 & $100.0 \%$ & 45 & $100.0 \%$ & 13 & $100.0 \%$ \\
\hline Mean (SD) & $1.9(6.0)$ & & $1.3(2.8)$ & & $1.0(1.4)$ & & $0.8(0.8)$ & \\
\hline Median (IQR) & $0.5(0.3-1.2)$ & & $0.5(0.3-0.7)$ & & $0.5(0.3-1.2)$ & & $0.5(0.3-1.2)$ & \\
\hline Total duration of therapy line, months ( $n$ ) & 203 & & 49 & & 45 & & 13 & \\
\hline \multicolumn{9}{|l|}{ Kaplan-Meier estimate } \\
\hline Median (95\% Cl) & $12.5(9.2-18.2)$ & & $7.9(5.8-11.2)$ & & $9.7(6.6-23.7)$ & & NE (6.1-NE) & \\
\hline Treatment ongoing ( $n, \%)$ & 73 & $36.0 \%$ & 16 & $32.70 \%$ & 15 & $33.3 \%$ & 9 & $69.20 \%$ \\
\hline $\begin{array}{l}\text { Number of patients who discontinued treatment } \\
(n, \%)\end{array}$ & 130 & $64.0 \%$ & 33 & $67.3 \%$ & 30 & $66.7 \%$ & 4 & $30.8 \%$ \\
\hline \multicolumn{9}{|l|}{ Reason for discontinuation $(n, \%)^{b, c}$} \\
\hline Adverse event & 3 & $2.3 \%$ & 0 & $0.0 \%$ & 1 & $3.3 \%$ & 0 & $0.0 \%$ \\
\hline Patient decision & 19 & $14.6 \%$ & 5 & $15.2 \%$ & 4 & $13.3 \%$ & 3 & $75.0 \%$ \\
\hline Progressive disease & 70 & $53.8 \%$ & 18 & $54.5 \%$ & 15 & $50.0 \%$ & 1 & $25.0 \%$ \\
\hline Completion of planned course of treatment & 43 & $33.1 \%$ & 9 & $27.3 \%$ & 11 & $36.7 \%$ & 1 & $25.0 \%$ \\
\hline Loss to follow-up & 3 & $2.3 \%$ & 0 & $0.0 \%$ & 0 & $0.0 \%$ & 0 & $0.0 \%$ \\
\hline Death & 9 & $6.9 \%$ & 2 & $6.1 \%$ & 2 & $6.7 \%$ & 0 & $0.0 \%$ \\
\hline Other: alternative treatment & 1 & $0.8 \%$ & 0 & $0.0 \%$ & 0 & $0.0 \%$ & 0 & $0.0 \%$ \\
\hline Unknown & 2 & $1.5 \%$ & 1 & $3.0 \%$ & 1 & $3.3 \%$ & 1 & $25.0 \%$ \\
\hline $\begin{array}{l}\text { Reasons for not administering additional cancer- } \\
\text { directed systemic treatment for advanced MTC, } \\
\text { among patients with no subsequent treat- } \\
\text { ment and patients who were alive at the time } \\
\text { of discontinuing last line of treatment }(n, \%)^{b}\end{array}$ & 72 & $35.5 \%$ & 27 & $55.1 \%$ & 15 & $33.3 \%$ & 3 & $23.1 \%$ \\
\hline Patient decision & 21 & $29.2 \%$ & 6 & $22.2 \%$ & 2 & $13.3 \%$ & 1 & $33.3 \%$ \\
\hline Frail physical status & 16 & $22.2 \%$ & 1 & $3.7 \%$ & 2 & $13.3 \%$ & 0 & $0.0 \%$ \\
\hline Stable disease & 23 & $31.9 \%$ & 5 & $18.5 \%$ & 8 & $53.3 \%$ & 1 & $33.3 \%$ \\
\hline
\end{tabular}

Cl confidence interval, ECOG Eastern Cooperative Oncology Group, IQR interquartile range, MTC medullary thyroid cancer, PS performance status, Q1 first quartile, Q3 third quartile, $R E T$ rearranged during transfection, $S D$ standard deviation

${ }^{\text {a }}$ Karnofsky score converted to the ECOG scale for 29 patients (14.3\%) [17]

${ }^{\mathrm{b}}$ Categories are not mutually exclusive. A patient may have had more than one reason/criterion assessed; thus, the column does not sum to $100 \%$

${ }^{\mathrm{c}}$ Among patients who discontinued treatment line

42 patients with known performance status at advanced MTC diagnosis, 36 (85.7\%) had a performance status of $0 / 1$.

Among patients with RET mutations, mean (SD) time from advanced diagnosis to initiation of first-line therapy was 1.0 (1.4) months (Table 4). Most ( $n=32 ; 71.1 \%)$ received only one line of therapy during the available follow-up time; 12 (26.7\%) received second-line therapy, and only $1(2.2 \%)$ received third-line therapy. Fifteen patients $(33.3 \%)$ were receiving ongoing first-line treatment at data abstraction. The mean (SD) number of lines of therapy received was $1.3(0.5)$; mean (SD) total duration of systemic therapy was 9.6 (10.2) months. The most common first-line therapies received were vandetanib $(n=18,40.0 \%)$, cabozantinib $(n=14,31.1 \%)$, and sorafenib $(n=5,11.1 \%)$. During first-line treatment, 28 patients (62.2\%) had an objective response; the median duration of objective response was not estimable for the 12 patients with known date of objective response. 


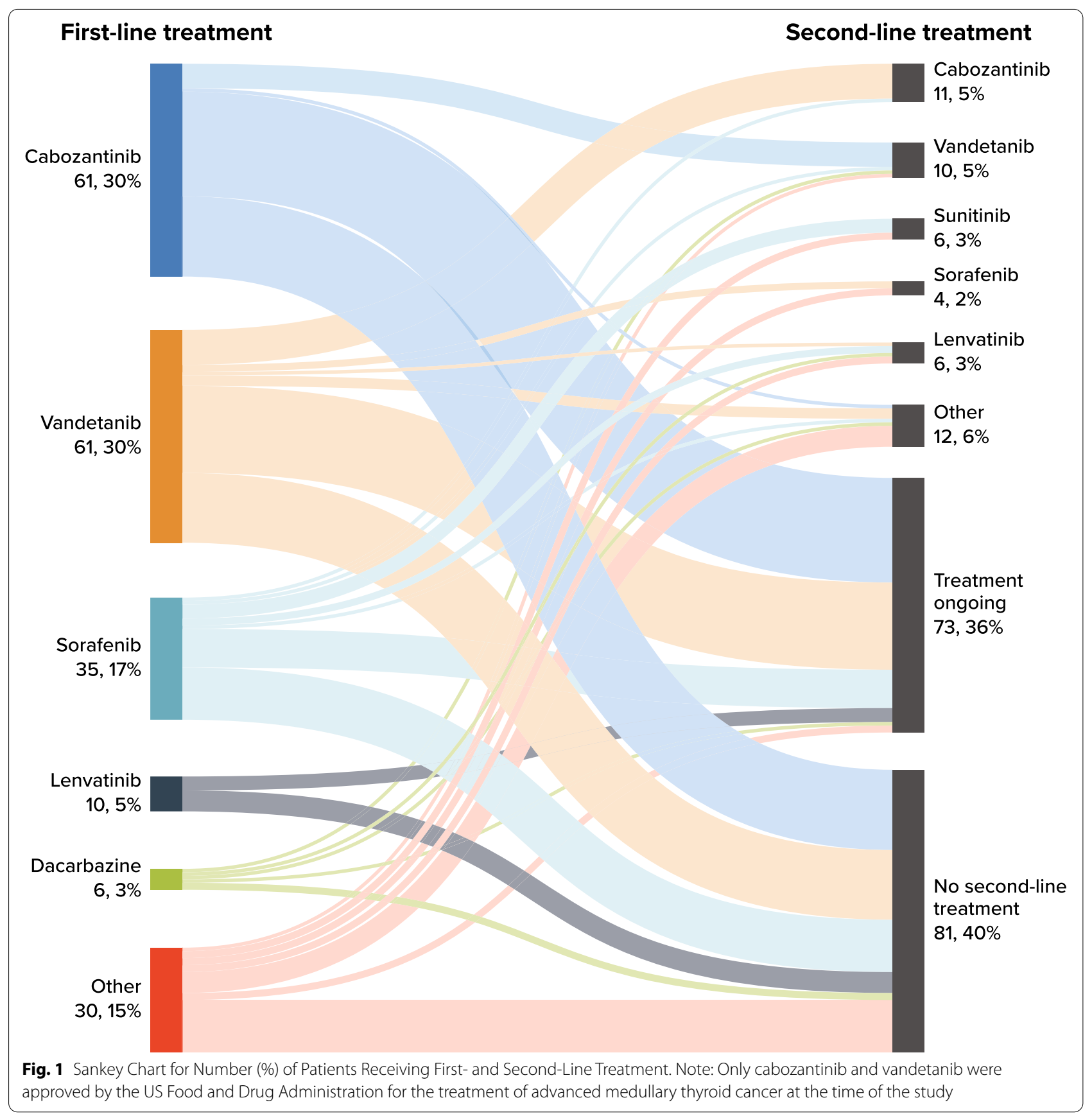

Median PFS for patients with RET-mutant MTC was 47.7 months (95\% CI, 14.2 months-not estimable) from initiation of first-line therapy and was not estimable from initiation of second-line therapy (Fig. 2B). Median OS was not estimable for these patients; survival rates at 12 , 36, and 60 months were $95.5 \%$ (95\% CI, 83.2\%98.9\%), 86.8\% (70.5\%-94.4\%), and 86.8\% (70.5\%-94.4\%), respectively.

\section{Patients undergoing second-line therapy}

Forty-nine patients in the overall study cohort (24.1\%) received second-line therapy during the available follow-up time. This subgroup had a mean (SD) age of 48.0 (10.5) years at advanced MTC diagnosis. At initiation of second-line therapy, performance status was reported for 35 patients, of whom 20 (57.1\%) had a performance status of $0 / 1$, and $15(42.9 \%)$ had a performance status of 2-4. The most frequent second-line treatments were 
Table 5 Objective Response Rate Among Patients With Advanced Medullary Thyroid Cancer

\begin{tabular}{|c|c|c|c|c|c|c|c|c|}
\hline \multirow[b]{3}{*}{ Objective response (complete or partial response) (n, \%) } & \multicolumn{4}{|c|}{ All Patients } & \multicolumn{4}{|c|}{ Patients With RET-Mutant MTC } \\
\hline & \multicolumn{2}{|c|}{$\begin{array}{l}\text { During First-Line } \\
\text { Therapy }\end{array}$} & \multicolumn{2}{|c|}{$\begin{array}{l}\text { During Second- } \\
\text { Line Therapy }\end{array}$} & \multicolumn{2}{|c|}{$\begin{array}{l}\text { During First-Line } \\
\text { Therapy }\end{array}$} & \multicolumn{2}{|c|}{$\begin{array}{l}\text { During } \\
\text { Second-Line } \\
\text { Therapy }\end{array}$} \\
\hline & 129 & $63.5 \%$ & 19 & $38.8 \%$ & 28 & $62.2 \%$ & 8 & $61.5 \%$ \\
\hline $\begin{array}{l}\text { Among those who had objective response and known date of } \\
\text { objective response, duration of response }(n)^{\text {a }}\end{array}$ & 71 & & 6 & & 12 & & 3 & \\
\hline Mean (SE) & $15.4(1.2)$ & & $16.8(-)$ & & $8.4(0.9)$ & & NE & \\
\hline Median & $\mathrm{NE}$ & & NE & & NE & & & \\
\hline $95 \% \mathrm{Cl}$ & 10.3 & NE & 16.8 & & 4.2 & NE & & \\
\hline Q1, Q3 & 6 & NE & 16.8 & & 6.6 & NE & & \\
\hline Censored (n, \%) & 46 & $64.8 \%$ & 5 & $83.3 \%$ & 7 & $58.3 \%$ & 3 & $100.0 \%$ \\
\hline
\end{tabular}

CI confidence interval, MTC medullary thyroid cancer, NE not estimable, Q1 first quartile, Q3 third quartile, RET rearranged during transfection, SE standard error

${ }^{a}$ Duration of objective response defined as time from complete or partial response to disease progression, death, or start of next line of treatment. Patients with treatment line ongoing or who discontinued treatment for nonprogression reasons were censored

cabozantinib ( $n=11,22.4 \%)$, vandetanib $(n=10,20.4 \%)$, lenvatinib and sunitinib ( $n=6[12.2 \%]$ for both), and sorafenib $(n=4,8.2 \%)$. During second-line treatment, 19 patients (38.8\%) had an objective response; the median duration of objective response was not estimable for the 6 patients with known date of objective response. During second-line therapy, regression modeling showed that available calcitonin levels remained generally stable (Fig. 4C). The number of available CEA levels was insufficient for analysis.

From initiation of second-line therapy, median PFS was 15.3 months (95\% CI, 6.6 months-not estimable) (Fig. 2C). Median OS was 22.4 months (95\% CI, 12.4 months-not estimable), and survival estimates at 12,36 , and 60 months from the initiation of second-line therapy were $69.1 \%$ (95\% CI, 52.8\%-80.8\%), 42.4\% (23.7\%$59.9 \%$ ), and $42.4 \%$ (23.7\%-59.9\%), respectively (Fig. 3B).

\section{Discussion}

This study retrospectively evaluated clinical characteristics, biomarkers, treatment patterns, and survival outcomes among US patients with advanced MTC managed in real-world clinical settings. Of the patients evaluated, $37.2 \%$ had RET-mutant MTC. Cabozantinib, vandetanib, sorafenib, and lenvatinib were the most common first-line treatments. Among 49 patients who received second-line treatment, most received cabozantinib, vandetanib, lenvatinib, or sunitinib. For the overall population, median PFS from start of first- and second-line treatments was 26.6 months and 15.3 months; median OS from initiation of first- and second-line treatments was 63.8 months and 22.4 months, respectively.

While real-world studies in MTC have been limited, particularly those evaluating treatment patterns, several recent studies have explored patient and tumor characteristics and treatment outcomes. Randle et al. [9] conducted a population-based study evaluating survival among patients with MTC (of all stages) using 2003-2012 data from the US SEER registry. Overall 5-year diseasespecific survival was estimated to be $51 \%$ among patients with metastatic MTC [9]. A higher 5-year disease-specific survival rate, $68.3 \%$, was observed for the overall population in the current study. This difference may be attributable to differences in the study time frames, potentially differing prognostic factors, and the introduction of new regimens since Randle and colleagues' analysis [9].

In addition, a registry study conducted in a routine care setting in Germany enrolled 48 patients with advanced MTC who were treated with the tyrosine kinase inhibitors (TKIs) vandetanib and/or cabozantinib [14]. This population had a median age at diagnosis of metastatic MTC of 50 years and predominantly had sporadic MTC (75\% of patients) $13 \%$ had hereditary MTC, and germline RET-mutation status was not known for $13 \%$ of patients. Most patients (96\%) had distant metastases. Twelvemonth survival estimates were $86 \%$ for those receiving vandetanib and $70 \%$ for those receiving cabozantinib [14]. The 12-month survival rate of $87.5 \%$ observed in the current study is consistent with this prior research, and median duration of treatment was similar in the two studies (25 months in Koehler et al. vs. 21.1 months in the current study). In addition, TKIs were the most commonly administered treatments in the current study: approximately $60 \%$ of patients received either cabozantinib or vandetanib in the first line. The TKIs sorafenib and lenvatinib, as well as the cytotoxic drug dacarbazine, were also commonly used in the first line, despite not being approved by the US Food and Drug Administration 
A

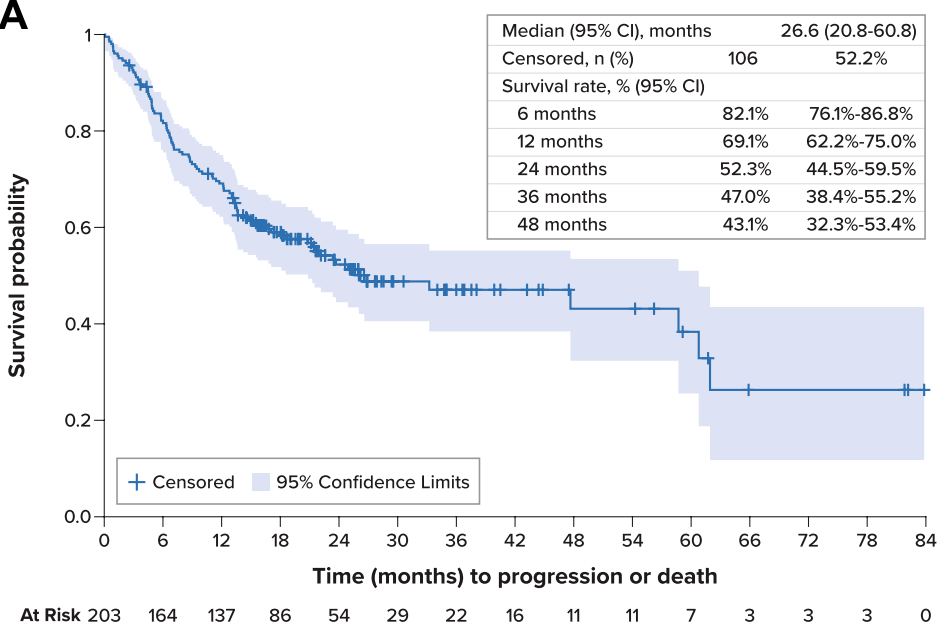

$\begin{array}{lllllllllllllll}\text { At Risk } 203 & 164 & 137 & 86 & 54 & 29 & 22 & 16 & 11 & 11 & 7 & 3 & 3 & 3 & 0\end{array}$
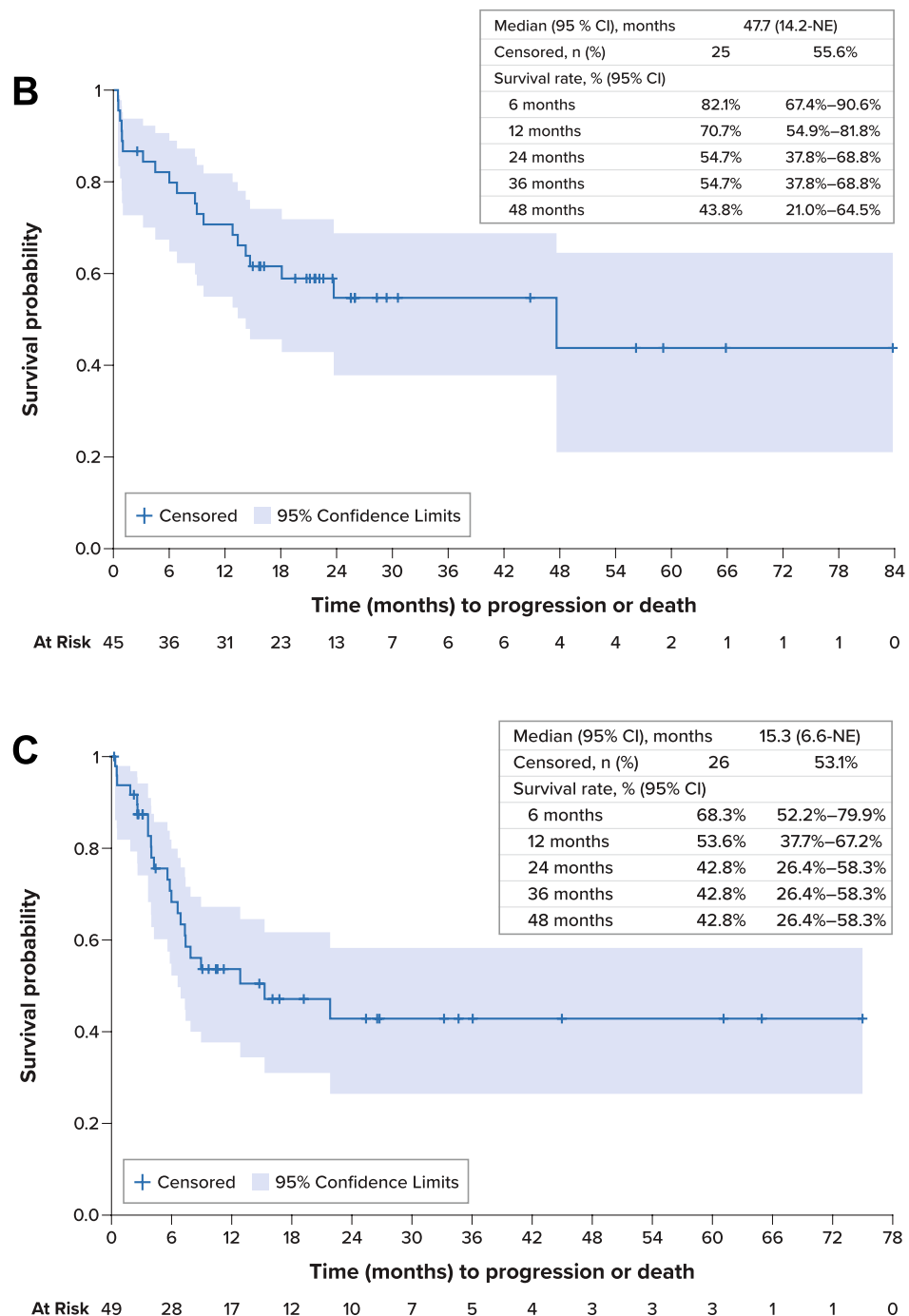

Fig. 2 Progression-Free Survival A. Overall Population, First-Line Therapy. B. RET-Mutant Medullary Thyroid Cancer, First-Line Therapy. C. Overall Population, Second-Line Therapy. Cl confidence interval, MTC medullary thyroid cancer, NE not estimable, PFS progression-free survival, RET rearranged during transfection, SE standard error 


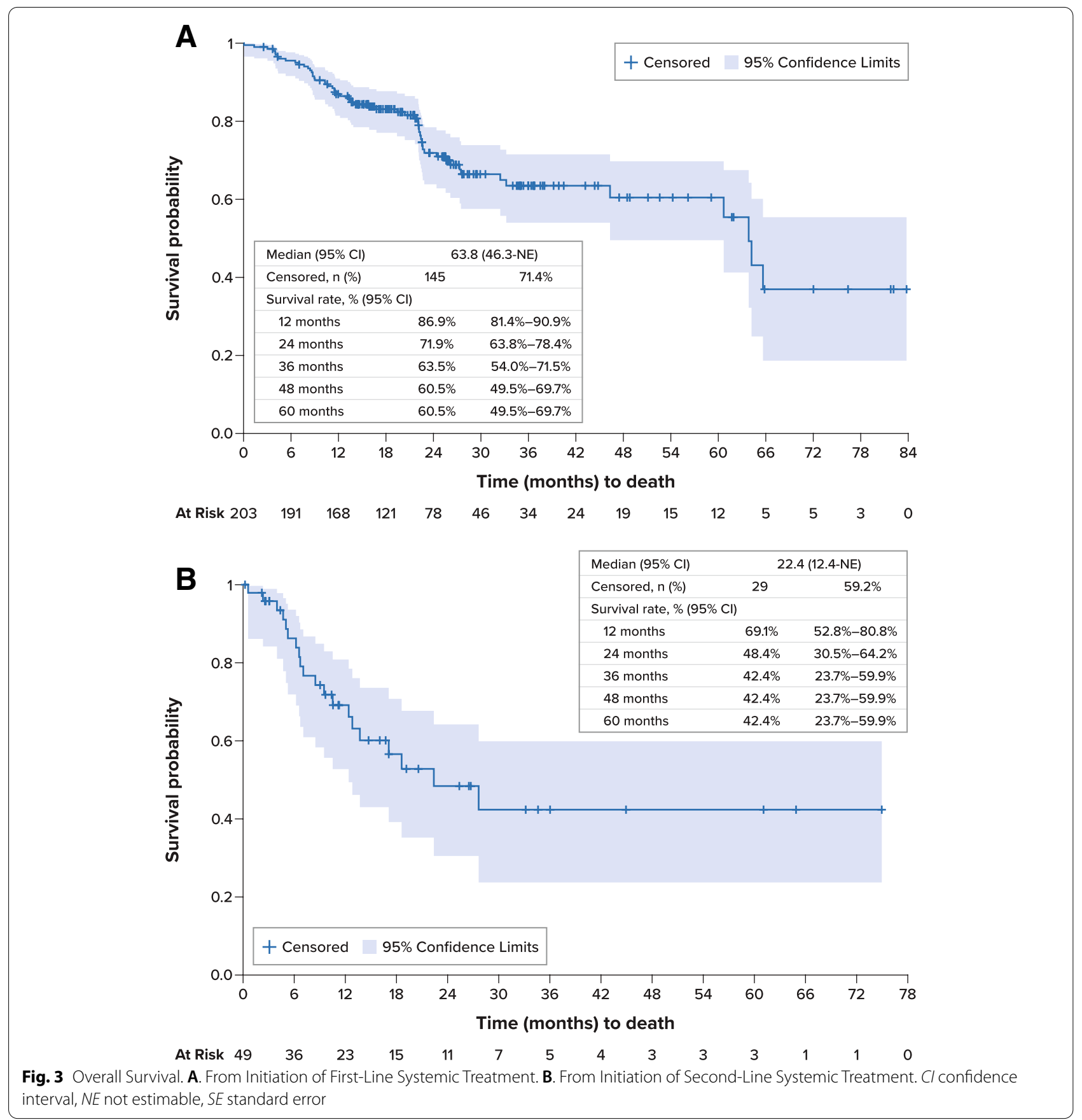

for the treatment of MTC. Presumably, treating physicians' off-label use of these therapies was driven by evidence of clinical benefit with sorafenib [19-22], lenvatinib [23], and dacarbazine [24, 25], as well as recommendations in clinical guidelines that small-molecule kinase inhibitors may be used when preferred systemic therapies are not available or appropriate [10].

RET-mutation positive MTC has been associated with worse clinical outcomes relative to MTC tumors that do not harbor $R E T$ mutations [5]. In clinical practice, the proportion of patients undergoing testing for RET mutations varies [26], and in the current study, $40 \%$ of patients were not known to have undergone testing for germline and/or somatic RET mutation. Among the overall sample, $22 \%$ of patients were known to have $R E T$-mutation positive MTC; these patients had an average age of 46.6 years. Vandetanib monotherapy was the most common first-line regimen for patients with $R E T$-mutation 

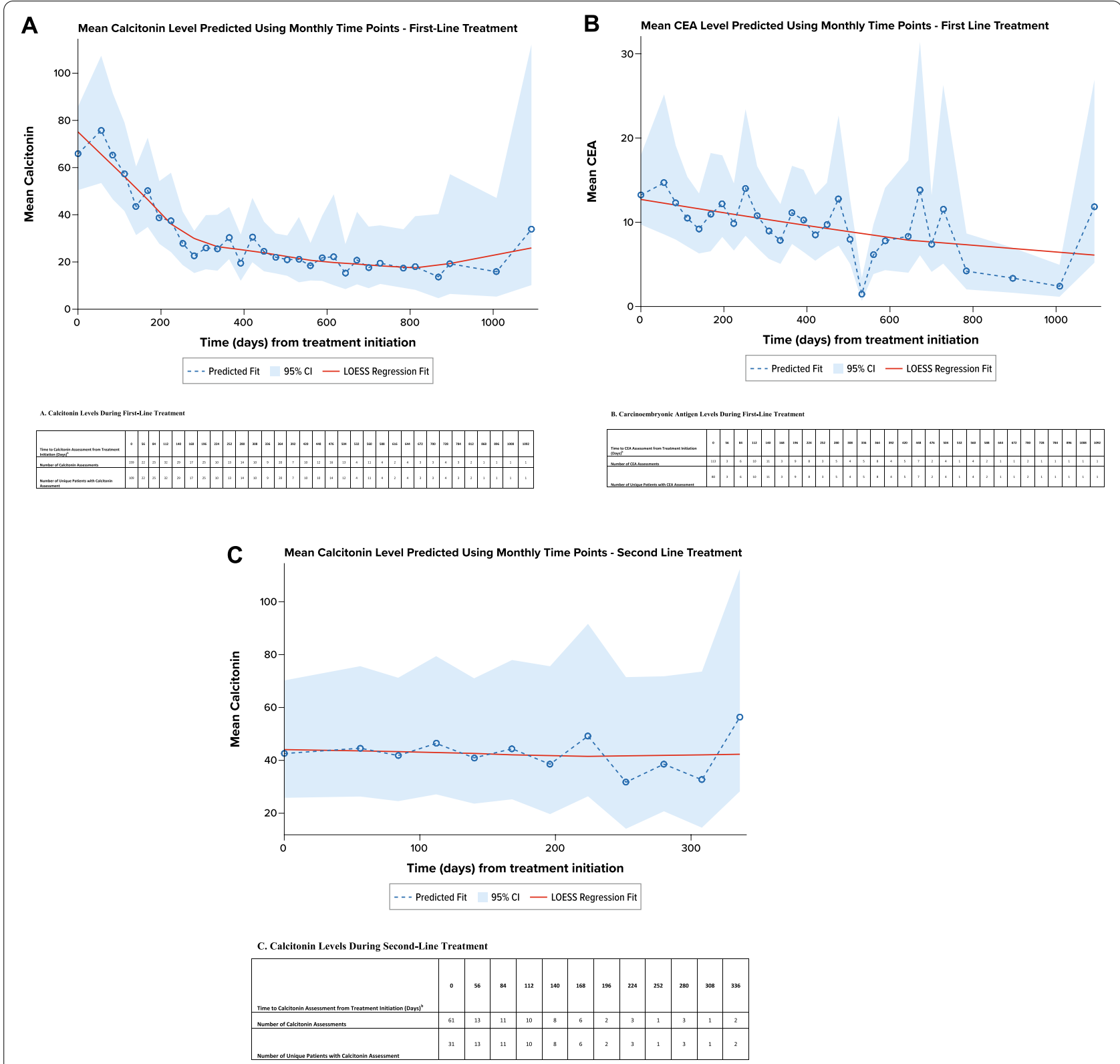

Fig. 4 Regression Analysis of Calcitonin and Carcinoembryonic Antigen Levels by Line of Treatment ${ }^{\mathrm{a}}$. A. Calcitonin Levels During First-Line Treatment. B. Carcinoembryonic Antigen Levels During First-Line Treatment. CEA carcinoembryonic antigen. C. Calcitonin Levels During Second-Line Treatment. CEA carcinoembryonic antigen. Note: A locally weighted polynomial regression, or LOESS, fit was estimated to smooth the

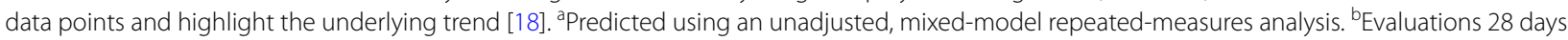
before or after initiating treatment line were attributed to baseline (i.e., time 0). Evaluation after 28 days of treatment initiation were grouped in to 28-day intervals and have been assigned to the end of the interval

positive MTC, followed by cabozantinib monotherapy. The PFS rate at 36 months was 55\% after initiation of first-line therapy for this subgroup. To date, studies evaluating real-world treatment patterns for patients with RET-mutation positive MTC have been limited, and future research should explore how treatment patterns and outcomes for patients with RET-mutant MTC evolve with the availability of the $R E T$-targeted therapies selpercatinib and pralsetinib.

Previous studies have found an association between elevated calcitonin and CEA levels, more rapid disease progression, and worse survival outcomes [13, 15]. A mixed-model repeated-measures regression analysis demonstrated that calcitonin levels decreased during 
first-line treatment initially; appeared to trend upward toward the end of first-line treatment, probably related to disease progression; and remained generally stable during second-line treatment. Because the objective response rate during second-line therapy (among the 49 patients who received it during this study) was lower $(39 \%)$ than that among all patients during first-line therapy (64\%), suboptimal response may potentially explain the pattern of calcitonin levels during second-line therapy, emphasizing the need for more effective treatments.

Several limitations of this study should be considered. Patients selected for study inclusion represent a convenience sample of medical records obtained from physicians willing to participate in the study and may be biased toward patients who were alive at data abstraction; study findings may not be generalizable to the overall population of US patients with advanced MTC. To help mitigate potential biases, physicians were recruited from a variety of regions and practice types and were instructed to select patients who were either alive or dead by a quasirandom procedure. Data available for study were limited to those recorded in medical records. Although internal data consistency was improved by data checks and use of a customized data-collection form, the entered data were not validated against patients' medical records by independent review. The information collected on genomic biomarker testing did not distinguish between germline and somatic mutations, and the frequency of such testing may have increased in current practice. Finally, a considerable proportion of patients were undergoing treatment at the time of data abstraction, and $52 \%$ of patients were censored for PFS estimates; therefore, PFS estimates are based on immature data, and studies with longer followup are warranted in this population.

\section{Conclusions}

More than one-third of patients with advanced or metastatic MTC were not tested for RET mutation as recommended by national guidelines. For the treatment of advanced/metastatic MTC, no specific preference of sequencing systemic agents was observed in the first- and second-line settings. The estimated OS was consistent with that observed from SEER data for metastatic MTC. Considering the recent approval of selective $R E T$ inhibitors for patients with $R E T$-mutant MTC, future research should investigate potential changes in these findings, particularly in the second-line setting. Evidence-based recommendation on sequencing of systemic therapies may benefit patients with advanced MTC.

\section{Abbreviations}

CEA: Cost-effectiveness analysis; Cl: Confidence interval; IRB: Institutional review board; MTC: Medullary thyroid cancer; NCCN: National Comprehensive
Cancer Network; OS: Overall survival; PFS: Progression-free survival; RET: Rearranged during transfection; SD: Standard deviation; SEER: Surveillance, Epidemiology, and End Results; TKI: Tyrosine kinase inhibitor; US: United States.

\section{Supplementary Information}

The online version contains supplementary material available at https://doi. org/10.1186/s13044-021-00119-9.

Additional file 1: Table S1. Most Common First- and Second-Line Systemic Therapies Among Patients With Advanced Medullary Thyroid Cancer.

\section{Acknowledgements}

Kate Lothman of RTI Health Solutions provided medical writing services during development of this manuscript. These services were funded by Eli Lilly and Company.

\section{Authors' contributions}

All authors made a significant contribution to the work reported, whether that is in the conception, study design, execution, acquisition of data, analysis and interpretation, or in all these areas; took part in drafting, revising, or critically reviewing the article; gave final approval of the version to be published; have agreed on the journal to which the article has been submitted; and agree to be accountable for the content of the article.

\section{Funding}

This study was performed under a research contract between RTI Health Solutions and Eli Lilly and Company and was funded by Eli Lilly and Company.

Availability of data and materials

Not applicable.

\section{Declarations}

\section{Ethics approval and consent to participate}

RTI International's institutional review board (IRB) reviewed the study protocol and deemed the research, which was not considered human subjects research, to be exempt from full IRB review.

\section{Consent for publication}

Not applicable.

\section{Competing interests}

This study was performed under a research contract between RTI Health Solutions and Eli Lilly and Company and was funded by Eli Lilly and Company. RP, EE, and JAK are employees of RTI Health Solutions. LMH and NRB are employees of Eli Lilly and Company.

\section{Author details}

${ }^{1}$ RTI Health Solutions, 3040 East Cornwallis Road, Research Triangle Park, NC 27709, USA. Eli Lilly and Company, Lilly Corporate Center, Indianapolis, IN 46285, USA. ${ }^{3}$ RTI Health Solutions, 307 Waverley Oaks Road, Waltham, MA 02452, USA.

Received: 21 June 2021 Accepted: 9 December 2021

Published online: 12 February 2022

\section{References}

1. Wells SA Jr, Asa SL, Dralle H, et al. Revised American Thyroid Association guidelines for the management of medullary thyroid carcinoma. Thyroid. 2015;25:567-610

2. Howlader N, Noone AM, Krapcho M, et al. (eds). SEER Cancer Statistics Review, 1975-2017, National Cancer Institute. Bethesda, MD. 2020 (based on November 2019 SEER data submission, posted to the SEER web site, 
April 2020). Available at https://seer.cancer.gov/csr/1975_2017/ Accessed 10 December 10, 2020.

3. Lim H, Devesa SS, Sosa JA, et al. Trends in thyroid cancer incidence and mortality in the United States, 1974-2013. JAMA. 2017;317:1338-48.

4. Moura MM, Cavaco BM, Leite V. RAS proto-oncogene in medullary thyroid carcinoma. Endocr Relat Cancer. 2015;22:R235-52.

5. Moura MM, Cavaco BM, Pinto AE, et al. Correlation of RET somatic mutations with clinicopathological features in sporadic medullary thyroid carcinomas. Br J Cancer. 2009;100:1777.

6. Larouche V, Akirov A, Thomas CM, et al. A primer on the genetics of medullary thyroid cancer. Curr Oncol. 2019;26:389-94.

7. Romei C, Ciampi R, Casella F, et al. RET mutation heterogeneity in primary advanced medullary thyroid cancers and their metastases. Oncotarget. 2018;9:9875.

8. Wells SA, Pacini F, Robinson BG, et al. Multiple endocrine neoplasia type 2 and familial medullary thyroid cancer: An update. J Clin Endocrinol Metab. 2013;98:3149-64.

9. Randle RW, Balentine CJ, Leverson GE, et al. Trends in the presentation, treatment, and survival of patients with medullary thyroid cancer over the past 30 years. Surgery. 2017;161:137-46.

10. National Comprehensive Cancer Network (NCCN). NCCN Clinical Practice Guidelines in Oncology (NCCN guidelines). Thyroid carcinoma, version 2. 2020. Available at https://www.nccn.org/store/login/login.aspx?Retur nURL=https://www.nccn.org/professionals/physician_gls/pdf/thyroid. pdf. Accessed 2 November 2, 2020

11. Al-Qurayshi Z, Khadra H, Chang K, et al. Risk and survival of patients with medullary thyroid cancer: National perspective. Oral Oncol. 2018;83:59-63.

12. Voss RK, Feng L, Lee JE, et al. Medullary thyroid carcinoma in MEN2A: ATA moderate- or high-risk RET mutations do not predict disease aggressiveness. J Clin Endocrinol Metab. 2017:102:2807-13.

13. Turkdogan S, Forest VI, Hier MP, et al. Carcinoembryonic antigen levels correlated with advanced disease in medullary thyroid cancer. J Otolaryngol Head Neck Surg. 2018;47:55.

14. Koehler VF, Adam P, Frank-Raue K, et al. Real-world efficacy and safety of cabozantinib and vandetanib in advanced medullary thyroid cancer. Thyroid. 2021;31:459-69.

15. Kotwal A, Erickson D, Geske J, et al. Predicting outcomes in sporadic and hereditary medullary thyroid carcinoma over two decades. Thyroid. 2021;31:616-26.

16. Detry MA, Ma Y. Analyzing repeated measurements using mixed models. JAMA. 2016:315(4):407-8.

17. Ma C, Bandukwala S, Burman D, et al. Interconversion of three measures of performance status: an empirical analysis. Eur J Cancer. 2010;46:3175-83.

18. Cleveland WS, Devlin SJ. Locally weighted regression: an approach to regression analysis by local fitting. J Am Statistical Assoc. 1988:83:596-610.

19. Capdevila J, Iglesias L, Halperin I, et al. Sorafenib in metastatic thyroid cancer. Endocr Relat Cancer. 2012;19:209-16.

20. Ahmed M, Barbachano Y, Riddell A, et al. Analysis of the efficacy and toxicity of sorafenib in thyroid cancer: a phase II study in a UK based population. Eur J Endocrinol. 2011;165:315-22.

21. Lam ET, Ringel MD, Kloos RT, et al. Phase II clinical trial of sorafenib in metastatic medullary thyroid cancer. J Clin Oncol. 2010;28:2323-30.

22. Thomas L, Lai SY, Dong W, Feng L, Dadu R, Regone RM, Cabanillas ME. Sorafenib in metastatic thyroid cancer: a systematic review. Oncologist. 2014:19:251-8.

23. Schlumberger M, Jarzab B, Cabanillas ME, et al. A phase II trial of the multitargeted tyrosine kinase inhibitor lenvatinib (E7080) in advanced medullary thyroid cancer. Clin Cancer Res. 2016;22:44-53.

24. Orlandi F, Caraci P, Berruti A, et al. Chemotherapy with dacarbazine and 5-fluorouracil in advanced medullary thyroid cancer. Ann Oncol. 1994:5:763-5.

25. Schlumberger M, Abdelmoumene N, Delisle MJ, Couette JE. Treatment of advanced medullary thyroid cancer with an alternating combination of 5 FU-streptozocin and 5 FU-dacarbazine. The Groupe d'Etude des Tumeurs a Calcitonine (GETC). Br J Cancer. 1995;71:363-5.

26. Mathiesen JS, Kroustrup JP, Vestergaard P, et al. Danish Thyroid Cancer Group (DATHYRCA). Completeness of RET testing in patients with medullary thyroid carcinoma in Denmark 1997-2013: A nationwide study. Clin Epidemiol. 2019;10:93-9.

\section{Publisher's Note}

Springer Nature remains neutral with regard to jurisdictional claims in published maps and institutional affiliations.
Ready to submit your research? Choose BMC and benefit from:

- fast, convenient online submission

- thorough peer review by experienced researchers in your field

- rapid publication on acceptance

- support for research data, including large and complex data types

- gold Open Access which fosters wider collaboration and increased citations

- maximum visibility for your research: over $100 \mathrm{M}$ website views per year

At BMC, research is always in progress.

Learn more biomedcentral.com/submissions 